Math. Model. Nat. Phenom.

Vol. 2, No. 2, 2007, pp. 40-55

\title{
On the Transition from Deflagration to Detonation in Narrow Channels
}

\author{
L. Kagan ${ }^{1}$ \\ Sackler Faculty of Exact Sciences, School of Mathematical Sciences \\ Tel Aviv University, Tel Aviv 69978, Israel
}

\begin{abstract}
A numerical study of a two-dimensional model for premixed gas combustion in a narrow, semi-infinite channel with no-slip boundary condition is performed. The work is motivated by recent theoretical advances revealing the major role of hydraulic resistance in deflagration-to-detonation transition, one of the central yet still inadequately understood phenomena of gaseous combustion. The work is a continuation and extension of recently reported results over non-isothermal boundary conditions, wider channels, and lower incipient flame velocities, closer to those of real life explosives.
\end{abstract}

Key words: computational fluid dynamics, detonation phenomena, transition from deflagration to detonation

AMS subject classification: $80 \mathrm{~A} 32,76 \mathrm{~L} 05$

\section{Introduction}

Spontaneous transition from deflagrative to detonative combustion, since its discovery more than a century ago by Bertholet and Vieille [1] and Mallard and LeChatelier [2], remains one of the central issues of premixed gas combustion, whose theoretical interpretation is still a matter of controversy. Turbulent combustion, experimentally observed and often considered to play a significant role in the transition, cannot be a pivotal cause because turbulent burning velocities are still a factor of 10 or more too small to generate pressure waves strong enough for triggering detonation. Recent theoretical findings based on the onedimensional ZND-Fanno model [3] suggest that the transition is presumably triggered by the

\footnotetext{
${ }^{1}$ Corresponding author. Email: kaganleo@post.tau.ac.il
} 
flow deceleration in the boundary layer, irrespective of whether the bulk flow is turbulent or not. Later numerical simulations in narrow channels [4-6], and experimental studies with capillaries [7] provide solid evidence that the transition may indeed take place in laminar flows.

The first numerical simulation of the transition in narrow channels was conducted under adiabatic conditions [4]. The impact of walls was accounted for through no-slip boundary conditions inducing resistance to the gas flow. The hydraulic resistance (friction) causes a gradual precompression and, hence, preheating of the fresh mixture adjacent to the advancing deflagration. After some induction period, this development leads to autoignition triggering an abrupt transition from deflagrative to detonative propagation. The detonation first develops in the boundary layer, where the impact of hydraulic resistance is stronger, and thereupon spreads over the channel's interior. The subsequent study of the problem [8] was aimed at elucidation of the impact of heat losses invariably present in realistic confined systems. The hydraulic resistance and heat losses exert opposite effects on the transition. The resistance raises the local temperature (through adiabatic compression) and thereby promotes autoignition. The heat loss tends to reverse this trend by reducing the temperature. In smooth channels, both mechanisms are of comparable influence. Therefore, one cannot be certain about the final outcome of the above competition. However, transition from deflagration to detonation is often observed experimentally.

As has been shown in [8], with the channel walls maintained at the ambient temperature, and the reaction kinetics assumed monomolecular, the transition does not occur, at least within the parameter range explored. However, for the bimolecular kinetics (other conditions as in the monomolecular case), the transition proves readily feasible, with the predetonation distance somewhat above that of the adiabatic limit. Higher molecularity implies higher sensitivity of the explosive mixture to the pressure change, which is quite significant in these problems.

The present study offers an extension of the previous one [8] over wider channels (100 flame-widths) and lower incipient flame velocities closer to those of real life explosives.

\section{Formulation}

To visualize the spatial picture of the transition, a wave of premixed gas combustion spreading from the closed to open end of the rectangular channel is studied by direct numerical simulations of the two dimensional Navier-Stokes equations for a compressible reactive flow.

The reaction rate is modeled by a single step Arrhenius kinetics. The latter is assumed to be of the first order with respect to the deficient reactant and of the 2-nd order with respect to the density, to account for the binary nature of chemical reaction taking place in real combustion systems.

In suitably chosen units the set of governing equations reads, 
continuity and state:

$$
\frac{\partial \hat{\rho}}{\partial \hat{t}}+\frac{\partial \hat{\rho} \hat{u}}{\partial \hat{x}}+\frac{\partial \hat{\rho} \hat{v}}{\partial \hat{y}}=0, \quad \hat{P}=\hat{\rho} \hat{T}
$$

momentum:

$$
\begin{aligned}
& \hat{\rho}\left(\frac{\partial \hat{u}}{\partial \hat{t}}+\hat{u} \frac{\partial \hat{u}}{\partial \hat{x}}+\hat{v} \frac{\partial \hat{u}}{\partial \hat{y}}\right)+\frac{1}{\gamma} \frac{\partial \hat{P}}{\partial \hat{x}}= \\
& \epsilon \operatorname{Pr}\left[2 \frac{\partial^{2} \hat{u}}{\partial \hat{x}^{2}}+\frac{\partial}{\partial \hat{y}}\left(\frac{\partial \hat{u}}{\partial \hat{y}}+\frac{\partial \hat{v}}{\partial \hat{x}}\right)-\frac{2}{3} \frac{\partial}{\partial \hat{x}}\left(\frac{\partial \hat{u}}{\partial \hat{x}}+\frac{\partial \hat{v}}{\partial \hat{y}}\right)\right], \\
& \hat{\rho}\left(\frac{\partial \hat{v}}{\partial \hat{t}}+\hat{u} \frac{\partial \hat{v}}{\partial \hat{x}}+\hat{v} \frac{\partial \hat{v}}{\partial \hat{y}}\right)+\frac{1}{\gamma} \frac{\partial \hat{P}}{\partial \hat{y}}= \\
& \epsilon \operatorname{Pr}\left[2 \frac{\partial^{2} \hat{v}}{\partial \hat{y}^{2}}+\frac{\partial}{\partial \hat{x}}\left(\frac{\partial \hat{u}}{\partial \hat{y}}+\frac{\partial \hat{v}}{\partial \hat{x}}\right)-\frac{2}{3} \frac{\partial}{\partial \hat{y}}\left(\frac{\partial \hat{u}}{\partial \hat{x}}+\frac{\partial \hat{v}}{\partial \hat{y}}\right)\right],
\end{aligned}
$$

heat:

$$
\begin{aligned}
\frac{1}{\gamma} \hat{\rho}\left(\frac{\partial \hat{T}}{\partial \hat{t}}+\hat{u} \frac{\partial \hat{T}}{\partial \hat{x}}+\hat{v} \frac{\partial \hat{T}}{\partial \hat{y}}\right)+\left(1-\frac{1}{\gamma}\right) \hat{P}\left(\frac{\partial \hat{u}}{\partial \hat{x}}+\frac{\partial \hat{v}}{\partial \hat{y}}\right)= \\
\epsilon\left(\frac{\partial^{2} \hat{T}}{\partial \hat{x}^{2}}+\frac{\partial^{2} \hat{T}}{\partial \hat{y}^{2}}\right)+(\gamma-1) \epsilon \operatorname{Pr} \hat{\Phi}+\left(1-\sigma_{p}\right) \hat{W}
\end{aligned}
$$

where

$$
\hat{\Phi}=2\left(\frac{\partial \hat{u}}{\partial \hat{x}}\right)^{2}+2\left(\frac{\partial \hat{v}}{\partial \hat{y}}\right)^{2}+\left(\frac{\partial \hat{v}}{\partial \hat{x}}+\frac{\partial \hat{u}}{\partial \hat{y}}\right)^{2}-\frac{2}{3}\left(\frac{\partial \hat{u}}{\partial \hat{x}}+\frac{\partial \hat{v}}{\partial \hat{y}}\right)^{2}
$$

concentration:

$$
\hat{\rho}\left(\frac{\partial \hat{C}}{\partial \hat{t}}+\hat{u} \frac{\partial \hat{C}}{\partial \hat{x}}+\hat{v} \frac{\partial \hat{C}}{\partial \hat{y}}\right)=\frac{\epsilon}{L e}\left(\frac{\partial^{2} \hat{C}}{\partial \hat{x}^{2}}+\frac{\partial^{2} \hat{C}}{\partial \hat{y}^{2}}\right)-\hat{W}
$$

chemical kinetics:

$$
\hat{W}=Z \hat{\rho}^{2} \hat{C} \exp \left(N_{p}\left(1-\hat{T}^{-1}\right)\right)
$$

Here $\hat{P}=P / P_{0}$ is the scaled pressure in units of the initial pressure, $P_{0} ; \hat{C}=C / C_{0}$, scaled concentration of the deficient reactant in units of its initial value, $C_{0} ; \hat{T}=T / T_{p}$, scaled temperature in units of $T_{p}=T_{0}+Q C_{0} / c_{p}$, adiabatic temperature of burned gas under constant pressure, $P_{0} ; T_{0}$ is the initial temperature of unburned gas; $Q$, heat release; $\sigma_{p}=T_{0} / T_{p} ; \gamma=c_{p} / c_{v} ; c_{p}, c_{v}$, specific heats; $\epsilon=\left(u_{p} / a_{p}\right)^{2}$, scaled thermal diffusivity, where 
$u_{p}$, velocity of the free-space (isobaric) deflagration relative to the burned gas is regarded as a prescribed parameter; $a_{p}=\sqrt{\gamma\left(c_{p}-c_{v}\right) T_{p}}, a_{0}=\sqrt{\gamma\left(c_{p}-c_{v}\right) T_{0}}$, velocities of sound at $T=T_{p}$ and $T=T_{0}$, respectively; $(\hat{u}, \hat{v})=(u, v) / a_{p}$, scaled flow velocity; $N_{p}=E / R T_{p}$, scaled activation energy; $\hat{\rho}=\rho / \rho_{p}$, where $\rho_{p}=P_{0} /\left(c_{p}-c_{v}\right) T_{p}$, density of the combustion products in free-space deflagration; $\hat{t}=t / t_{p},(\hat{x}, \hat{y})=(x, y) / x_{p}, x_{p}=a_{p} t_{p}$, where $t_{p}=A^{-1} Z \exp \left(N_{p}\right)$ is the reference time; $Z=\frac{1}{2} L e^{-1} N_{p}^{2}\left(1-\sigma_{p}\right)^{2}$ is the normalizing factor to ensure that at $N_{p}>>1$ and adiabatic free-space conditions the scaled deflagration velocity relative to the burned gas is close to $\sqrt{\epsilon} ; A$, pre-exponential factor; $\operatorname{Pr}, L e$ - are the Prandtl and Lewis numbers, respectively. In the adopted formulation the molecular transport coefficients as well as specific heats are assumed to be constant.

As may be readily shown, $x_{p}=a_{p} t_{p}=l_{t h} / \sqrt{\epsilon}$, where $l_{t h}=D_{t h}^{0} / u_{0}$ is the flame width, $D_{t h}^{0}$, thermal diffusivity at $T=T_{0}$ and $u_{0}=\sigma_{p} u_{p}$, velocity of the free-space deflagration relative to the fresh mixture.

Eqs (2.1)-(2.7) are considered in the strip $0<\hat{x}<\infty, 0<\hat{y}<\hat{d} / 2$, corresponding to the upper half of a semi-infinite channel, and under the following boundary and initial conditions:

closed end of the channel:

$$
\partial \hat{T}(0, \hat{y}, \hat{t}) / \partial \hat{x}=0, \quad \partial \hat{C}(0, \hat{y}, \hat{t}) / \partial \hat{x}=0, \quad \hat{u}(0, \hat{y}, \hat{t})=0, \quad \hat{v}(0, \hat{y}, \hat{t})=0,
$$

far end of the channel:

$$
\begin{gathered}
\hat{T}(+\infty, \hat{y}, \hat{t})=\sigma_{p}, \quad \hat{C}(+\infty, \hat{y}, \hat{t})=1, \quad \hat{u}(+\infty, \hat{y}, \hat{t})=0 \\
\hat{v}(+\infty, \hat{y}, \hat{t})=0, \quad \hat{\rho}(+\infty, \hat{y}, \hat{t})=1 / \sigma_{p} .
\end{gathered}
$$

heat conducting wall:

$$
\partial \hat{T}(\hat{x}, \hat{d} / 2, \hat{t}) / \partial \hat{y}=-B i(T-\sigma), \quad \partial \hat{C}(\hat{x}, \hat{d} / 2, \hat{t}) / \partial \hat{y}=0, \quad \hat{u}(\hat{x}, \hat{d} / 2, \hat{t})=0, \quad \hat{v}(\hat{x}, \hat{d} / 2, \hat{t})=0,
$$

where $B i$ is the Biot number, symmetry plane:

$$
\partial \hat{T}(\hat{x}, 0, \hat{t}) / \partial \hat{y}=0, \quad \partial \hat{C}(\hat{x}, 0, \hat{t}) / \partial \hat{y}=0, \quad \partial \hat{u}(\hat{x}, 0, \hat{t}) / \partial \hat{y}=0, \quad \hat{v}(\hat{x}, 0, \hat{t})=0,
$$

initial conditions:

$$
\begin{gathered}
\hat{T}(\hat{x}, \hat{y}, 0)=\sigma_{p}+\left(1-\sigma_{p}\right) \exp (-\hat{x} / \hat{l}), \quad \hat{C}(\hat{x}, \hat{y}, 0)=1 \\
\hat{P}(\hat{x}, \hat{y}, 0)=1, \quad \hat{u}(\hat{x}, \hat{y}, 0)=0, \quad \hat{v}(\hat{x}, \hat{y}, 0)=0
\end{gathered}
$$

Here $\hat{l}$ is the scaled width of the initiation hot spot.

To elucidate the impact of heat losses the simulations were conducted for adiabatic $(B i=$ $0)$, as well as non-adiabatic $(B i>0)$ boundary conditions. 


\section{Numerical simulations}

To avoid too large a disparity between the spatio-temporal scales involved, the numerical simulations are conducted for somewhat reduced value of the inverse Mach number $a_{p} / u_{p}$, compared to that typical of real-life explosives. Specifically, we set:

$$
\begin{gathered}
N_{p}=4 ; \quad a_{p} / u_{p}=10 ; \quad \sigma_{p}=0.2 ; \quad L e=1 ; \\
\operatorname{Pr}=1 ; \quad \gamma=1.3 ; \quad \hat{l}=0.25 ; \quad \hat{d}=2,10 .
\end{gathered}
$$

For the chosen parameter set the channel widths specified as $20 l_{t h}$ and $100 l_{t h}$; the flame burning velocity $u_{0}$ is $\sqrt{\epsilon \sigma_{p}}=0.045$-times the velocity of sound $a_{0}$.

The computational method used and its validation are described in [4]. The resolution tests are presented in [8] and the Appendix.

Figure 1 depicts the reaction front profiles at several consecutive instants of the time. The configurations shown correspond to isothermal $(B i=\infty)(a)(c)$ and adiabatic $(B i=0)$ $(b)(d)$ boundary conditions, with channel widths $d=10(a)(b)$ and $d=2(c)(d)$. Similarly to the results reported in [8], shortly after the ignition but when details of the initiation are already forgotten the flame assumes a $U$ - shape convex towards the fresh mixture and flattened near the center. Thereupon the flame generally develops two bulges near the channel walls (Fig.1 $(a)(b)(d)$ ). The mechanism responsible for the bulging is discussed in [9]. The bulges grow and eventually merge at the centerline of wide channels. The merging induces secondary shocks and velocity spikes clearly seen on Figs. 2,3 and 4,5.

The latter effect has not been observed in our previous study [8] where the transition occurred prior to the merging. In narrow cold-walled channels the bulges do not form, due to the shortage of space for their development (Fig. 1(c)) Figures 2-7 show a sequence of tonal images of gradient norms $|\nabla \hat{P}|$. The conditions employed are identical to those of Fig. 1. Here one can readily identify the evolving flame, leading shock, secondary shocks, emergence of the detonation and retonation waves, as well as transverse shocks induced by the shock-wall interaction. In all cases considered, both adiabatic and non-adiabatic, the incipient detonation emerges at the most advanced parts of the flame front - simultaneously at two points for the flame front with two maxima (Figs 2,3 and 7) and at the channel centerline for the front with a single maximum (Fig. 6). ${ }^{2}$ The explosion takes place directly on the flame front, triggering detonation and retonation waves.

Prior to its settling into the Chapman-Jouguet regime, the detonation passes through the fresh mixture precompressed and preheated by the precursor shock, which results in the propagation velocity overshoot (Figs 6,7). Note the islands of unburned gas formed near the wall immediately after the autoignition event (Fig. 1). Figure 8 plots the temporal evolution of the reaction wave velocity along the channel's axis calculated for several channel widths $(d)$ at isothermal boundary conditions. As one would anticipate, the predetonation distance is longer for wider channels. The transition fails in sufficiently narrow channels

\footnotetext{
${ }^{2}$ This picture differs from that of the monomolecular kinetics where in the adiabatic case the detonation is conceived directly on the channel's wall at the trailing part of the flame [4].
} 

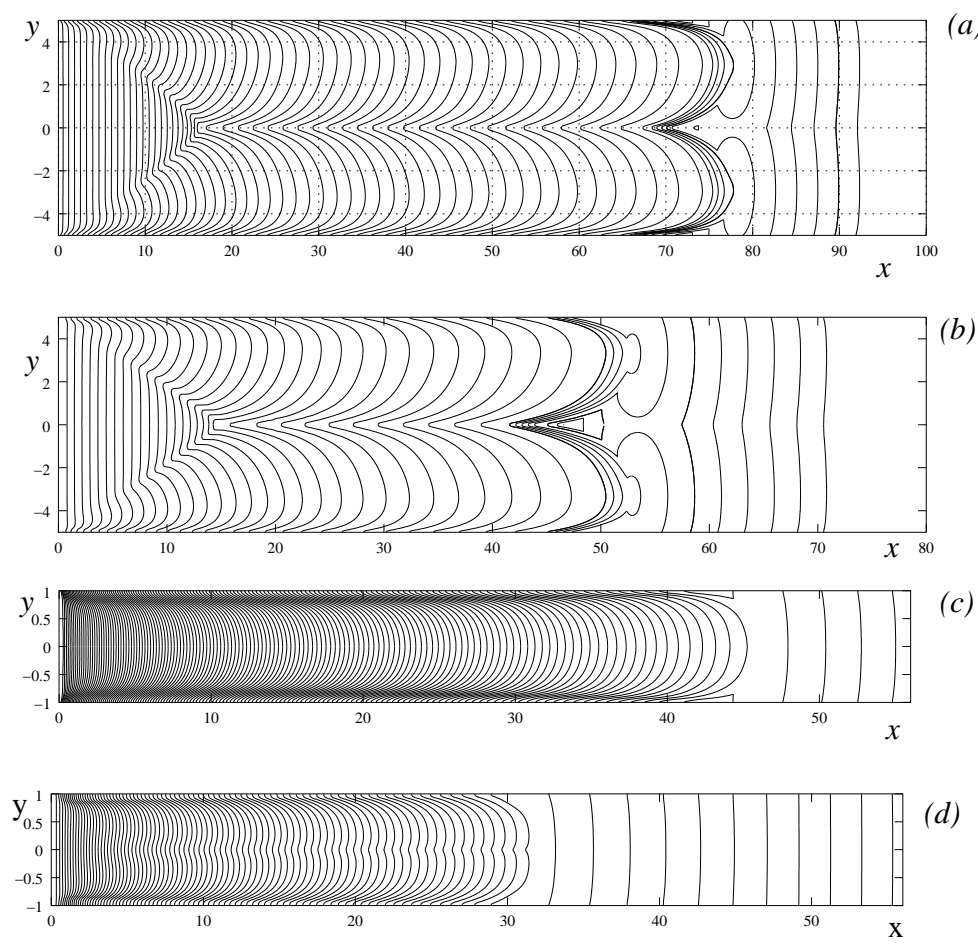

Figure 1: Temporal evolution of the reaction front. Scaled coordinates $(x, y)$ are in units of $10 l_{t h}$. The parameters employed are specified as: $N=4, L e=1, \operatorname{Pr}=1, \epsilon=0.01$, $\sigma=0.2, \gamma=1.3, d=10(a)(b)$ and $d=2(c)(d)$.Figures $(a)(c)$ - correspond to isothermal walls $(B i=\infty)$, and $(b)(d)$ to adiabatic walls $(B i=0)$. Figures $(c)(d)$ depict the reaction front profiles at several equidistant moments of time. In Figures $(a)(b)$ time intervals are equally spaced only up to the transition point, above which the intervals are subjected to the 5 -fold reduction. 

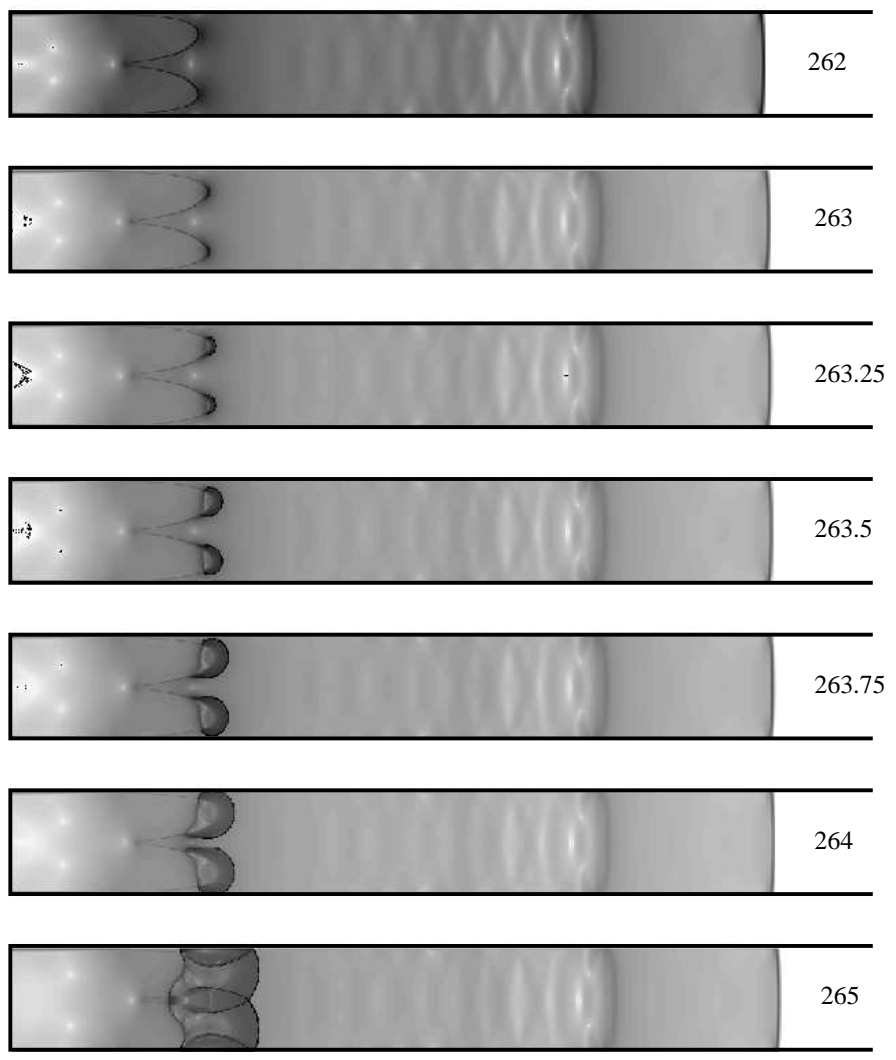

Figure 2: Pressure gradient norm at several consecutive instants of time (marked on the right). Stronger shading corresponds to higher pressure gradient. Parameters are identical to those of Fig. $1 a$. 

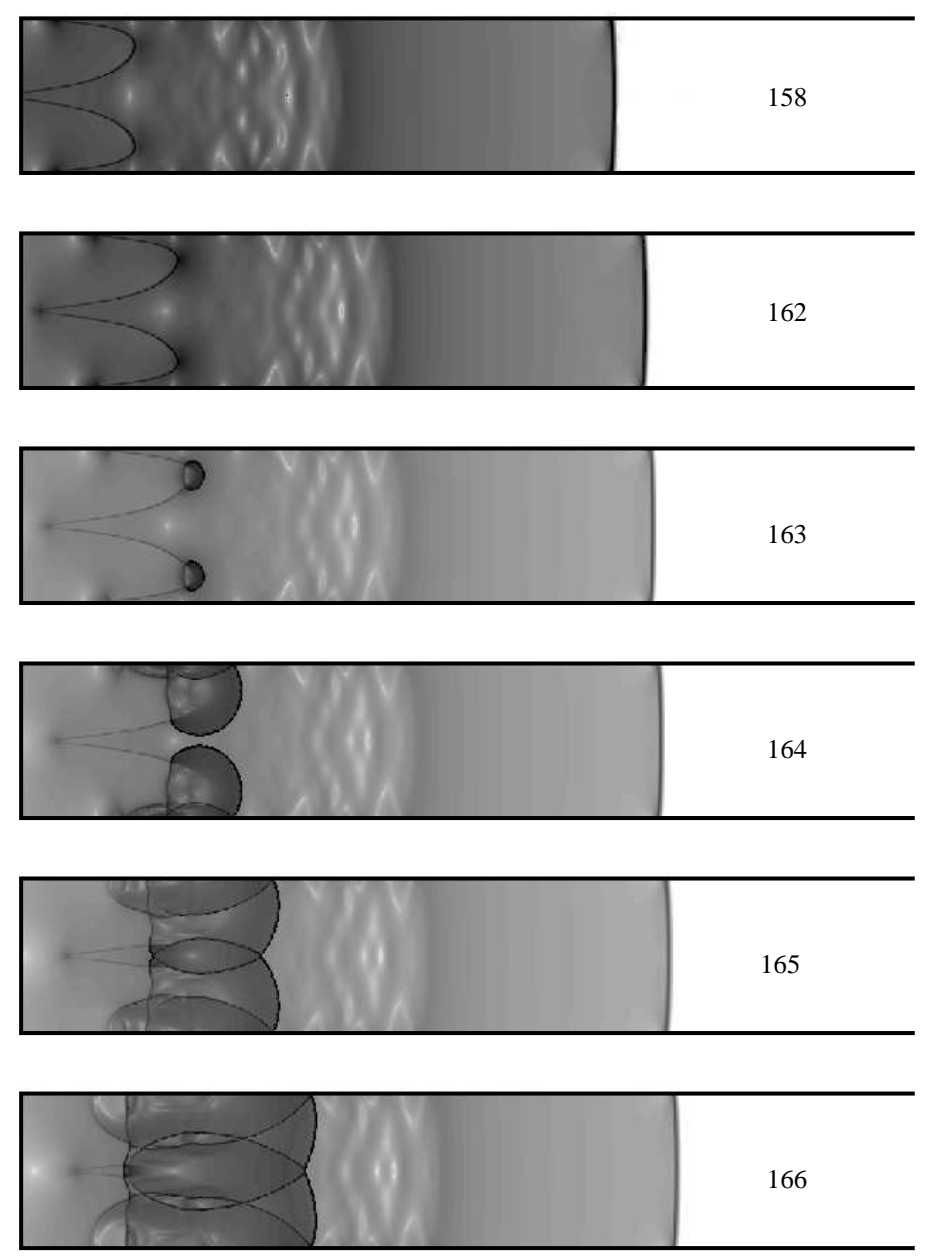

Figure 3: Pressure gradient norm at several consecutive instants of time (marked on the right). Stronger shading corresponds to higher pressure gradient. Parameters are identical to those of Fig. $1 b$. 


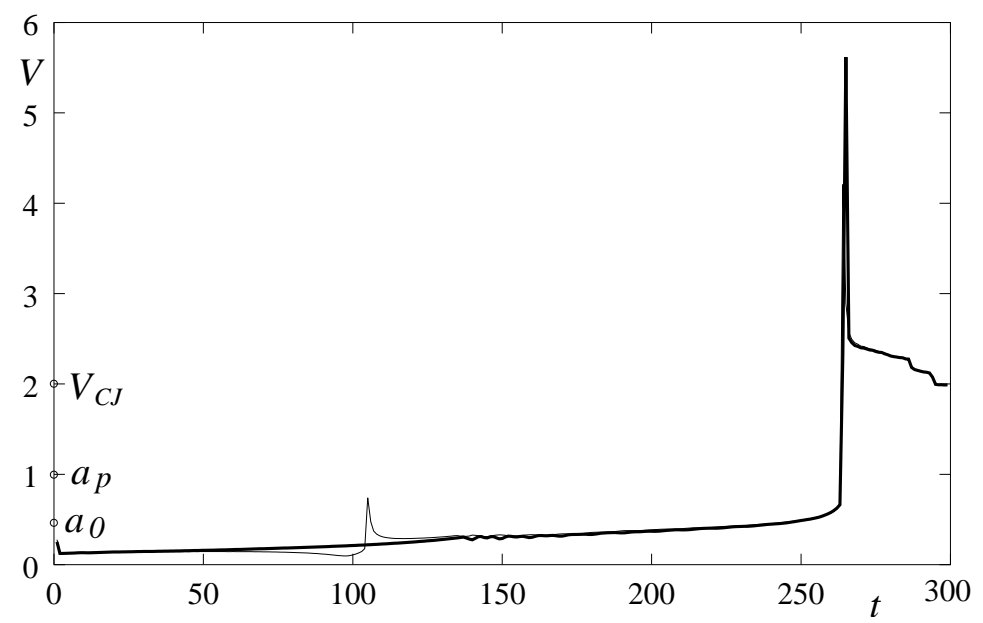

Figure 4: Reaction wave velocity $V$ (scaled) versus time $t$ (scaled). $V_{C J}$ corresponds to the Chapman-Jouget detonation; $a_{u}, a_{b}$ - velocities of sound in the unburned and burned mixture, respectively. The bold/thin line correspond to $y=4.5 / 1$, respectively. Parameters are identical to those of Fig. $1 a$

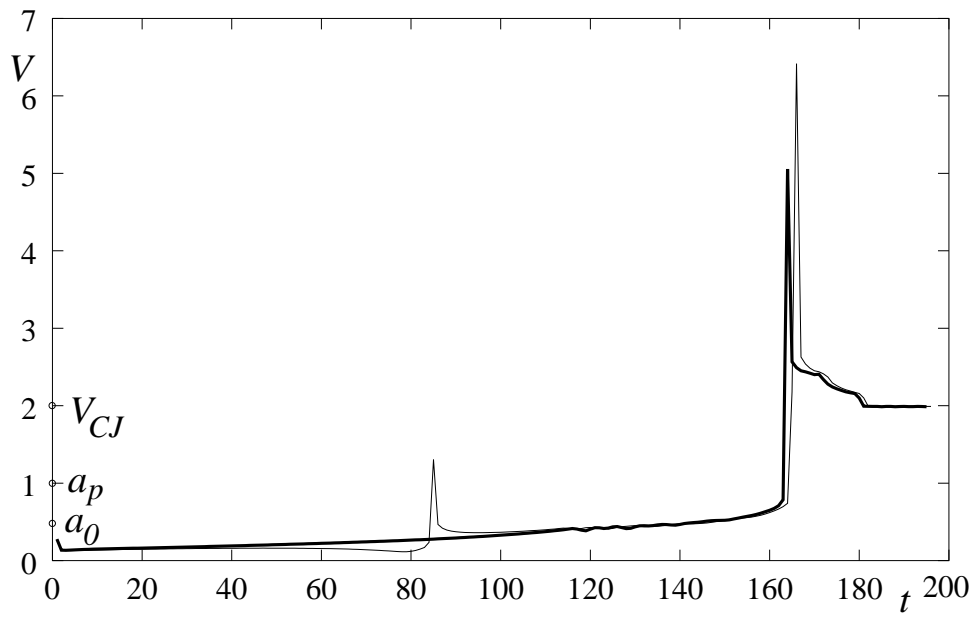

Figure 5: Reaction wave velocity $V$ (scaled) versus time $t$ (scaled). $V_{C J}$ corresponds to the Chapman-Jouget detonation; $a_{u}, a_{b}$ - velocities of sound in the unburned and burned mixture, respectively. The bold/thin line correspond to $y=4.5 / 1$, respectively. Parameters are identical to those of Fig. $1 b$ 


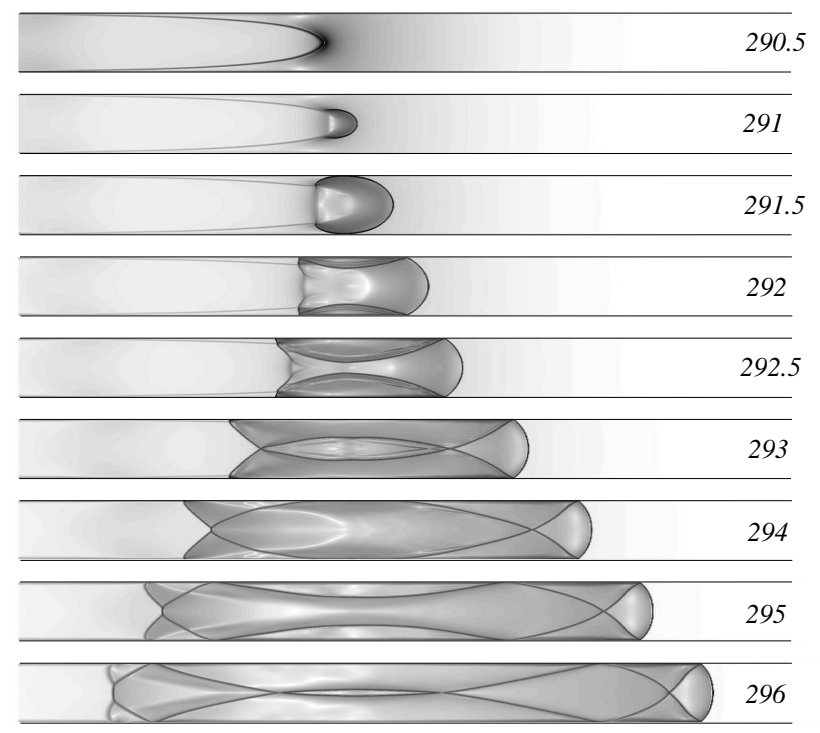

Figure 6: Pressure gradient norm at several consecutive instants of time (marked on the right). Stronger shading corresponds to higher pressure gradient. Parameters are identical to those of Fig. $1 c$.

$\left(d<d_{c r}=0.6\right)$ resulting in total extinction rather than settling into the deflagrative mode. A similar observation has been reported in the recent experimental study of the transition in capillaries [7].

Note that $d_{c r}=0.6$ is actually not a detonability limit. For the chosen parameter set one may well generate a steady self-sustained detonation at $d>0.35$, provided the initial hot spot is wide enough (direct initiation). Yet, under the no-slip boundary conditions the steady deflagration does not seem feasible, at least in the parameter space explored.

The above simulations deal with two extreme cases of $B i=0$ and $B i=\infty$. Figure 9 shows the temporal evolution of the combustion wave speed calculated for several intermediate Biot numbers at $d=0.4$. As one would anticipate, the increasing of the Biot number results in longer predetonation times and distances. Moreover, there is a critical Biot number above which the transition fails altogether. Similarly to the isothermal case the failure of the transition does not result in a deflagrative propagation but rather in the total extinction.

\section{Concluding remarks}

The results obtained are qualitatively similar to those of [8] for faster flames, reinforcing the argument in favor of the friction based concept of the transition. The inability to induce a self-sustained deflagration may be attributed to the particular form and parameters of the reaction rate adopted. The numerous data on detonability vs. flammability limits in narrow tubes provide solid evidence that the long-lived deflagrative propagation is indeed feasible. The flammability limits are generally wider than the detonability limits. However 


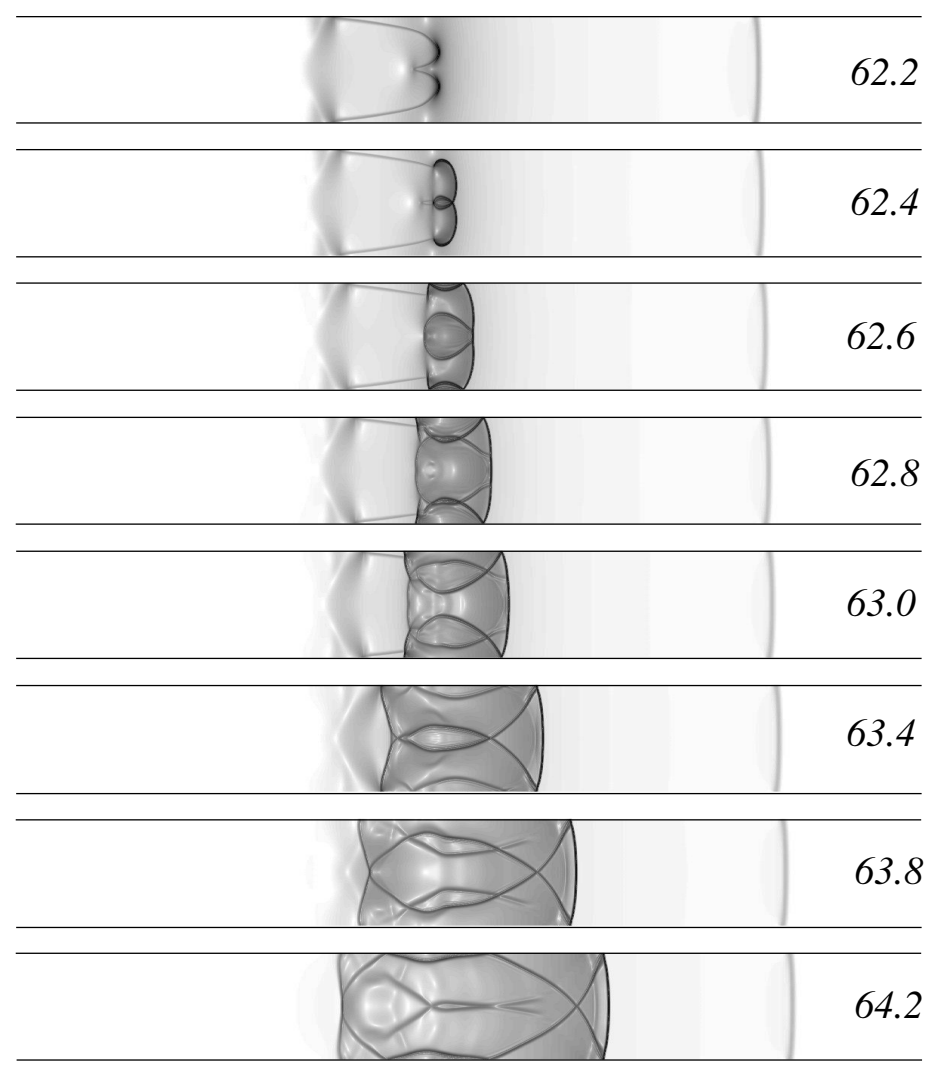

Figure 7: Pressure gradient norm at several consecutive instants of time (marked on the right). Stronger shading corresponds to higher pressure gradient. Parameters are identical to those of Fig. $1 b$.

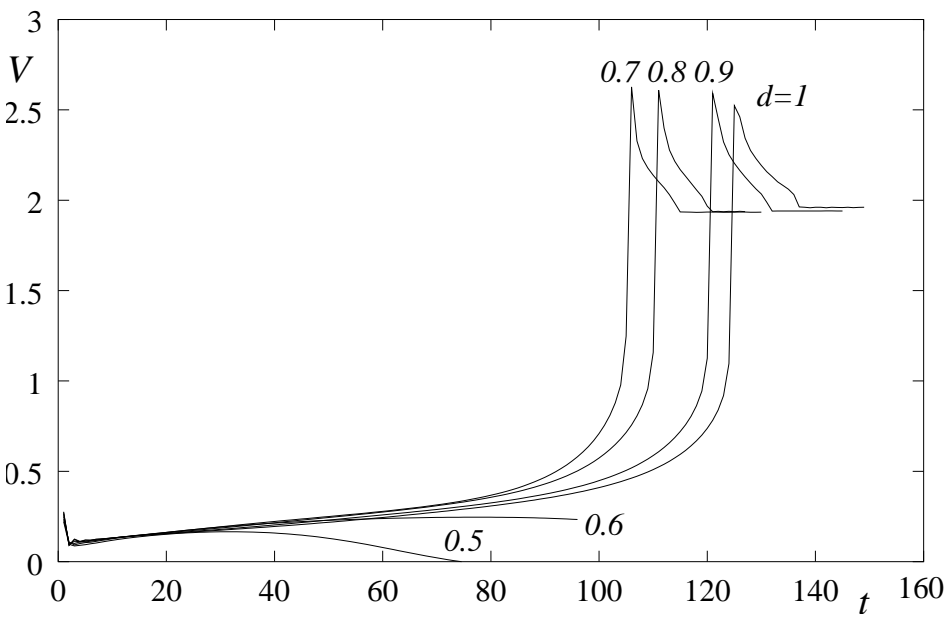

Figure 8: Reaction wave velocity $V$ (scaled) versus time $t$ (scaled) for several channel widths, $d$, at isothermal boundary conditions. 


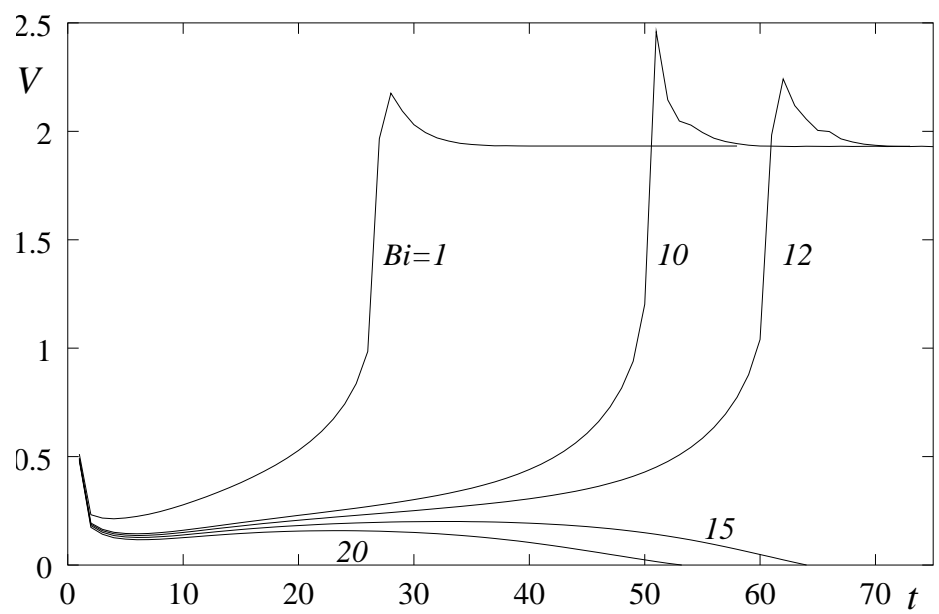

Figure 9: Reaction wave velocity $V$ (scaled) versus time $t$ (scaled) for several Biot numbers, $B i ; d=0.4$.

at relatively low activation energies the situation may reverse [10], and this seems to be the case in the present study. More research on this fundamental and intriguing question is needed.

For bimolecular kinetics and adiabatic boundary conditions the hot spot triggering the transition emerges at a certain distance from the channel's wall rather than at the wall itself, as occurs for monomolecular kinetics [4]. It would be interesting to see what happens in wider channels - whether the initiation hot spot stays close to the wall, in accordance with experimental observations [11], or moves deeper into the channel's interior.

Finally, we would like to report on a recently found alternative mechanism of the transition, unrelated to the friction [6]. It transpires that in wide enough channels where, due to the Darrieus-Landau instability, the flame interface becomes wrinkled, the transition may be triggered by autoignition of the fresh mixture trapped within one of the flame folds. Under normal conditions this effect should be feasible in fast burning systems such as acetylenefluorine mixtures where the normal flame-speed may rise as high as $20 \mathrm{~m} / \mathrm{s}$. Yet, for many conventional explosives this would require an appropriate preconditioning (preheating, precompression) to make the flame-induced autoignition possible. The difficulty in reaching the autoignition in moderately fast burning mixtures perhaps explains why the transition is reluctant to occur in unconfined system dealing with outward propagating 'free-space' flames.

Acknowledgments These studies were supported by the U.S.-Israel Binational Science Foundation (Grant 2006-151), the Israel Science Foundation (Grant 350/05) and the European Community Program RTN-HPRN-CT-2002-00274. The author thanks Vadim Gamezo, Michael Liberman, Elaine Oran, Gregory Sivashinsky and Damir Valiev for helpful discussions. 


\section{References}

[1] M. Bertholet, P. Vieille. Sur la vitesse de propagation des phenomenes explosifs dans les gas. C. R. Acad, Sci. Paris, 1882, Vol. 94, 101-108.

[2] E. Mallard, H. LeChatelier. Recherches sur la combustion des mélanges gazeux explosifs. Annales des Mines, 1883, Vol. 4, 274-568.

[3] I. Brailovsky. G. Sivashinsky. Hydraulic resistance as a mechanism for deflagration-todetonation transition. Combust. Flames, 2000, Vol. 122, 492-499.

[4] L. Kagan, G. Sivashinsky. The transition from deflagration to detonation in thin channels. Combust. Flames, 2003, Vol. 134, 389-397.

[5] E.S. Oran, V.N. Gamezo. Flame acceleration and detonation transition in narrow tubes. 2005, Proc. 20 ${ }^{\text {th }}$ ICDERS , Montreal, Canada (CD)

[6] M.A. Liberman, G.I. Sivashinsky, D.H. Valiev, L.-E. Eriksson. Numerical simulation of deflagration-to-detonation transition: role of hydrodynamic instability. Int. J. Tran. Phenomena, 2006, Vol. 8, 253-277.

[7] M.-h. Wu, M.P. Burke, S.F. Son, R.A. Yetter. Flame acceleration and the transition yo detonation of stoichiometric ethylene/oxygen in microscale tubes. Proc. Combust. Inst. 2006, Vol. 31, 2429-2436

[8] L. Kagan, D. Valiev, M. Liberman, V. Gamezo, E. Oran, and Sivashinsky, G. Effect of hydraulic resistance and heat losses on the deflagration-to-detonation transition. In Pulsed Detonation Engine, Ed. S. Frolov, Torus Press Ltd, Moscow, 2006, 51-62.

[9] J.D. Ott, E.S. Oran, J.D. Anderson. The interaction of a flame and its self-induced boundary layer. NASA-Report, 1999, CR-1999-209401.

[10] V. Bykov, I. Goldfarb, V. Gol'dshtein, L. Kagan, G. Sivashinsky. Effect of hydraulic resistance and heat losses on detonability and flammability limits. Combust. Theory Modelling, 2004, Vol. 8, 413-424.

[11] K.K. Kuo Principles of Combustion, John Wiley \& Sons, 2005.

[12] S.V. Patancar, Numerical Heat Transfer and Fluid Flow, Taylor \& Francis, 1980.

[13] S. Xu, T. Aslam, D.S. Stewart, High resolution numerical simulation of ideal and nonideal compressible reacting flows with embedded internal boundaries. Combust. Theory Modelling, 1997, Vol. 1,113-142. 


\section{Appendix. Resolution tests}

The above formulated problem is solved numerically by employing an explicit finitedifference technique with the 'upwind' approximation for convective terms and the central differences for the other spatial derivatives [12]. The boundary conditions are implemented by the method of 'ghost' points [13]. Resolution tests are similar to those conducted in [8] for twice higher flame velocity.
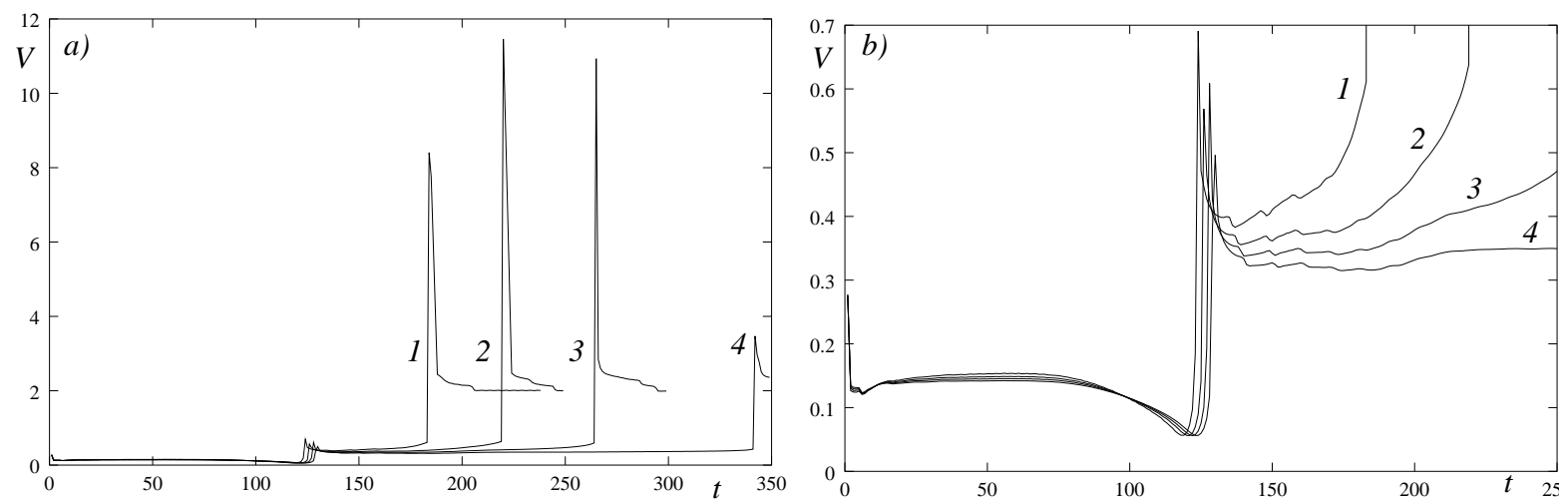

Figure 10: Reaction wave velocity $V$ (scaled) versus time $t$ (scaled) for several resolutions at isothermal boundary conditions $(d=10)$.
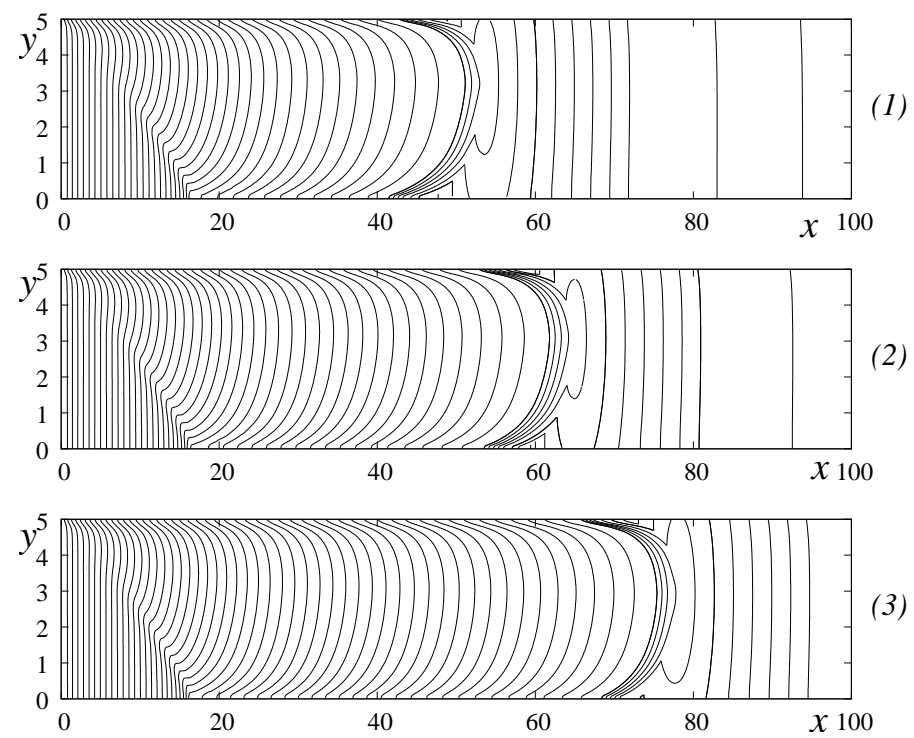

Figure 11: Front configurations for several consecutive instants of times at different resolutions. (Table 1, 1-3). Note the disparity between the transversed and longitudinal scales and reduction of the time intervals above the transition point.

Figures 10, 11 and Table 1 show results of the test calculated for a wide channel $(d=$ 10) with isothermal boundary conditions $(B i=\infty)$. The dynamical picture of the 


\begin{tabular}{c|cccccc}
\hline & $\Delta x$ & $\Delta y$ & $t_{D D T}$ & $\Delta t_{D D T}$ & $l_{D D T}$ & $\Delta l_{D D T}$ \\
\hline 1 & 0.0148 & 0.0148 & 184 & & 51.6 & \\
2 & 0.0111 & 0.0111 & 220 & 36 & 67.4 & 15.8 \\
3 & 0.00833 & 0.00833 & 265 & 45 & 81.6 & 14.2 \\
4 & 0.00555 & 0.00555 & 342 & 77 & 94.1 & 12.5 \\
\hline
\end{tabular}

Table 1: Data on resolution tests for $d=10 . \Delta x, \Delta y$ are the spatial steps; $t_{D D T}, l_{D D T}, \Delta t_{D D T}, \Delta l_{D D T}$ are the predetonation times and distances, and their increments.

DDT event may be divided into three zones: pre-DDT, proper DDT, and past-DDT. As is readily seen, there is a fairly good convergence of the $V(t)$ curves (Fig. 10) in the pre-DDT and post-DDT zones, that is prior to the bulge merging and upon the transition where the detonation settles into the Chapman-Jouguet regime. There is however no convergence in the intermediate, proper DDT, zone (Table 1). Unfortunately, employment of finer grids leads to prohibitively long time runs. In spite of the lack of convergence in the DDT zone there is a qualitative similarity between the numerical solutions, which leads one to believe that the latter are not computational artifacts but a truthful, albeit qualitative, reflection of the principal features of the original system.

According to Table 1 the relative increments corresponding to the most resolved simulations are,

$$
\frac{\Delta t_{D D T}}{t_{D D T}}=22.5 \% \quad \frac{\Delta l_{D D T}}{l_{D D T}}=13.3 \%
$$

That is, the calculated predetonation times and distances are still far from their true values.

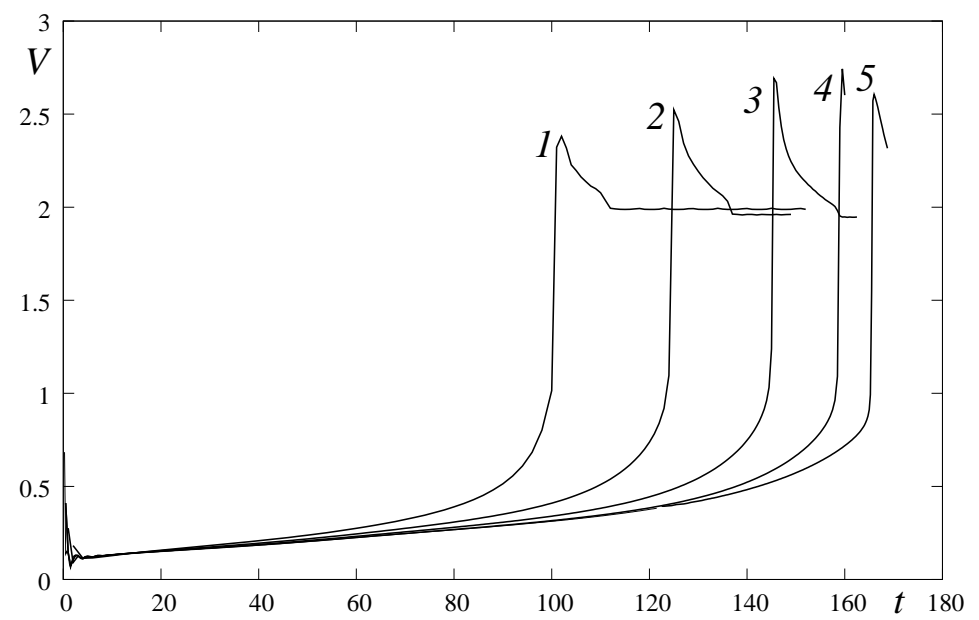

Figure 12: Reaction wave velocity $V$ (scaled) versus time $t$ (scaled) at different resolutions (Table 2, 1-5) 


\begin{tabular}{c|cccccc}
\hline & $\Delta x$ & $\Delta y$ & $t_{D D T}$ & $\Delta t_{D D T}$ & $l_{D D T}$ & $\Delta l_{D D T}$ \\
\hline 1 & 0.0125 & 0.0222 & 101 & & 31.1 & \\
2 & 0.00625 & 0.0111 & 125 & 24 & 40.3 & 11.2 \\
3 & 0.00313 & 0.0055 & 145 & 20 & 46.6 & 6.3 \\
4 & 0.00156 & 0.00275 & 159.5 & 14.5 & 52.2 & 5.6 \\
5 & 0.00078 & 0.00138 & 165.8 & 6.3 & 53.2 & 1.0 \\
\hline
\end{tabular}

Table 2: Data on resolution tests for $d=2$. See also the legend for Table 1 .

Figure 12 and Table 2 show results of the resolution tests conducted $\mathrm{f}$ or a narrow $(d=2)$ adiabatic channel $(B i=0)$. In this case the convergence does take place.

The relative increments corresponding to the most resolved simulations are,

$$
\frac{\Delta t_{D D T}}{t_{D D T}}=3.7 \% \quad \frac{\Delta l_{D D T}}{l_{D D T}}=1.9 \%,
$$

indicating a clear tendency towards convergence. 